\title{
Connexin43 dephosphorylation at serine 282 is associated with connexin43-mediated cardiomyocyte apoptosis
}

\author{
Yutong Yang ${ }^{1} \cdot$ Xinxin Yan ${ }^{1} \cdot$ Jingyi Xue ${ }^{1} \cdot$ Yuanyuan Zheng $^{1} \cdot$ Min Chen $^{1} \cdot$ Zhipeng Sun $^{1} \cdot$ Tiantian Liu $^{1}$ • \\ Cong Wang ${ }^{1} \cdot$ Hongjie You ${ }^{1} \cdot$ Dali Luo ${ }^{1}$
}

Received: 4 September 2018 / Revised: 2 January 2019 / Accepted: 4 January 2019 / Published online: 15 February 2019

(c) ADMC Associazione Differenziamento e Morte Cellulare 2019

\begin{abstract}
Gap junction protein connexin 43 (Cx43) plays an important role in regulating cardiomyocyte survival in addition to regulating electrical coordination. $\mathrm{Cx} 43$ dephosphorylation, found in severe cardiac pathologies, is thought to contribute to myocardial injury. However, the mechanisms underlying $\mathrm{Cx} 43$ mediation of cell survival and myocardial lesions remain unknown. Here, we found that transfecting an adenovirus carrying a mutant gene of Cx43-serine 282 substituted with alanine (S282A) into neonatal rat ventricular myocytes (NRVMs) induced cell apoptosis and $\mathrm{Ca}^{2+}$ transient desynchronization, whereas using gap junction inhibitor or knocking down $\mathrm{Cx} 43$ expression with Cx43-miRNA caused uncoupled $\mathrm{Ca}^{2+}$ signaling without cell death. Similarly, while $\mathrm{Cx} 43-\mathrm{S} 282 \mathrm{~A}^{+/+}$failed in generation, $\mathrm{Cx} 43-\mathrm{S} 282 \mathrm{~A}^{+/-}$mice exhibited cardiomyocyte apoptosis and ventricular arrhythmias dependent on S282 dephosphorylation. Further, Cx43 dephosphorylation at S282 activated p38 mitogen-activated protein kinase (p38 MAPK), factor-associated suicide and the caspase- 8 apoptotic pathway by physically interacting with p38 MAPK. These findings uncovered a specific Cx43 phosphorylation residue involved in regulating cardiomyocyte homeostasis. S282 phosphorylation deficiency acts as a trigger inducing cardiomyocyte apoptosis and cardiac arrhythmias, providing a potential mechanism for Cx43-mediated myocardial injury in severe cardiac diseases.
\end{abstract}

\section{Introduction}

Cardiomyocyte apoptosis is a fundamental cause of myocardial injury and cardiac dysfunction in severe diseases. Several stimuli, including reactive oxygen species generation, autoimmune response, cell cycle perturbation and ATP consumption, are proposed to be associated with activating the cardiomyocyte death machinery [1-3]. Evidence has

These authors contributed equally: Yutong Yang, Xinxin Yan

Edited by S. Lavandero.

Supplementary information The online version of this article (https:// doi.org/10.1038/s41418-019-0277-x) contains supplementary material, which is available to authorized users.

Dali Luo

luodl@ccmu.edu.cn

1 Department of Pharmacology, Beijing Key Laboratory of Metabolic Disturbance Related Cardiovascular Disease, School of Basic Medical Sciences, Capital Medical University, Beijing 100069, P.R. China also demonstrated that the gap channel protein, connexin 43 (Cx43), plays an important role in regulating cell survival and death processes and participating in severe myocardial lesions [3-6].

$\mathrm{Cx} 43$ is the major connexin protein in ventricular gap junctions. Because $\mathrm{Cx} 43$ plays a central role in cardiac electrical coupling and development, $C x 43$ gene mutations in humans are associated with severe arrhythmias, oculodentodigital dysplasia, visceroatrial heterotaxia, and sudden infant death [7-12]. Mice with a complete Cx43 knockout die as neonates $[10,11]$, while heart-specific $\mathrm{Cx} 43$-deficient mice (approximately 90\% ablation) and heterozygous Cx43truncated or site-mutated mice reach adulthood with unchanged cardiac structure and function. However, these mice have enhanced risks of spontaneous and inducible ventricular tachyarrhythmias and sudden death [7, 9, 12]. Therefore, cardiac electrical disturbances and aberrant development are the dominant phenotypes of $C x 43$ gene-alterationassociated human diseases and animal models. In addition, posttranscriptional modulations, particularly phosphorylation modifications, significantly alter $\mathrm{Cx} 43$ function and biotransformation. Normal myocardial $\mathrm{Cx} 43$ is highly 
a
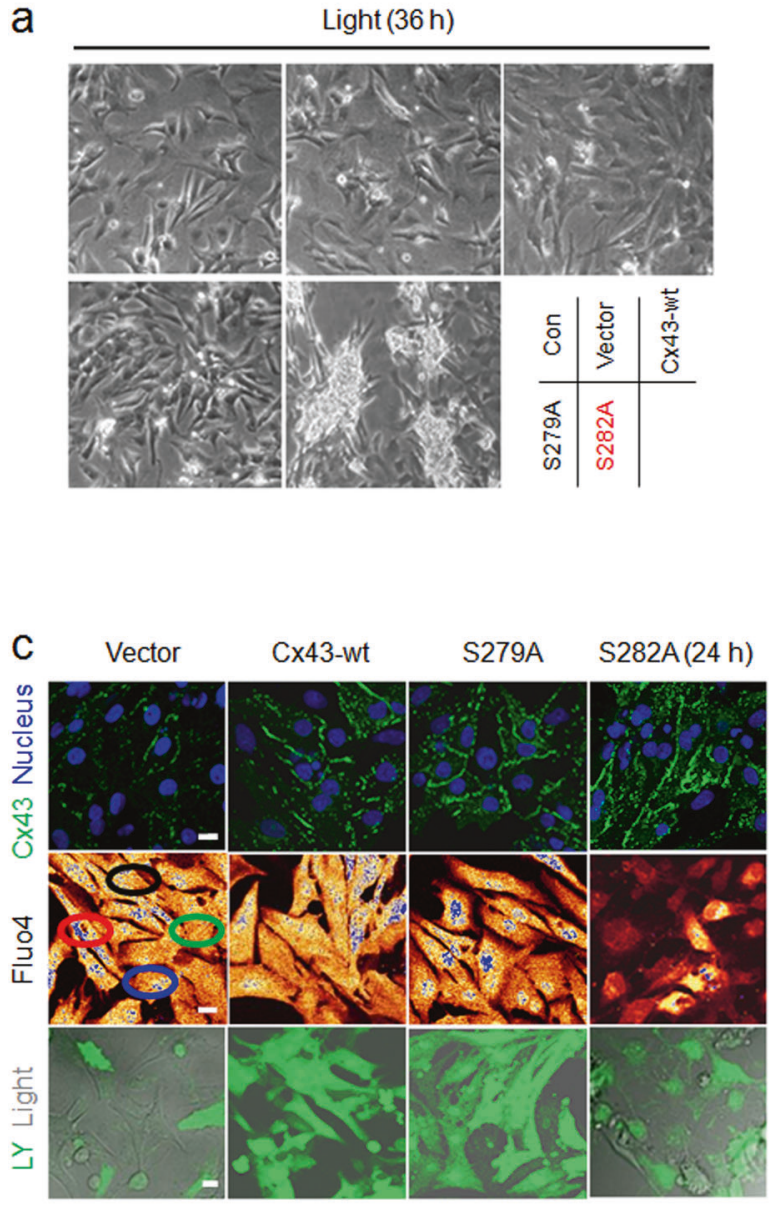

b

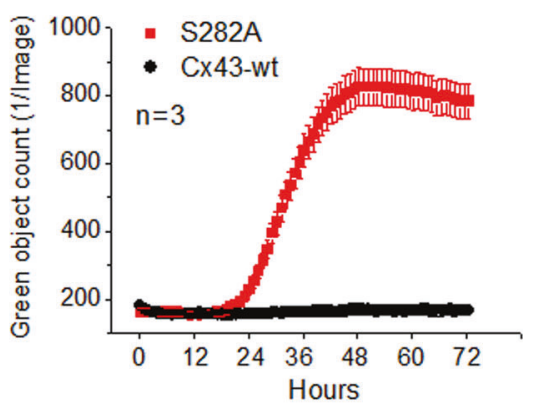

d

$$
\mathrm{Ca}^{2+} \text { signal }
$$

Vector $(n=5) \quad$ Cx43-wt (5) S279A (5) S282A (6)
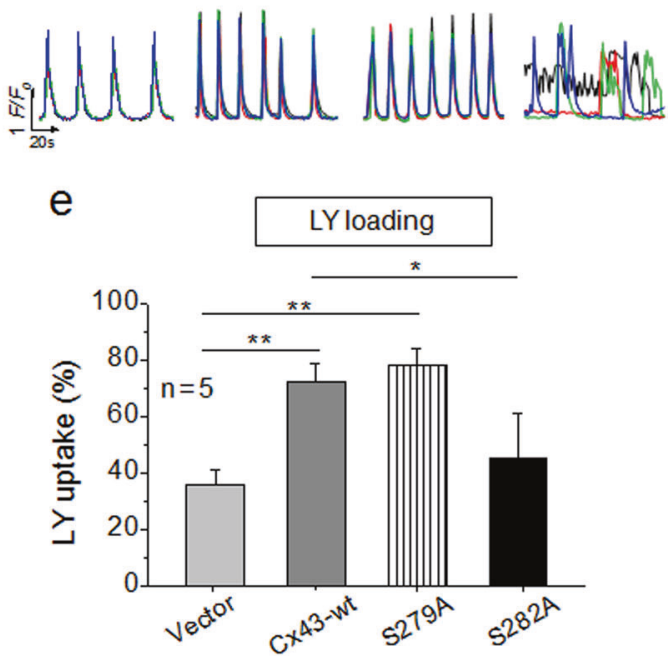

images of NRVMs transfected with different mutants for $24 \mathrm{~h}$, and then stained with antibody against $\mathrm{Cx} 43$, or loaded with Fluo4 to detect $\mathrm{Ca}^{2+}$ transients or Lucifer Yellow (LY) to detect intercellular communication, as indicated (c). Nucleus was stained with Hochest33258. Scale bar: $10 \mu \mathrm{m}$. Typical traces of $\mathrm{Ca}^{2+}$ transients illustrate that S282A cells had uncoupled transients (d) and decreased LY uptake (e). $* P<0.05, * * P<0.01$, unpaired two-tailed Student's $t$ test, $n=4-6$ independent determinations for each group as indicated

(Fas)/Fas-associating protein with a novel death domain (FADD) pathway and induces cardiomyocyte apoptosis. Moreover, by physically interacting with $\mathrm{Cx} 43$, p38 mitogenactivated protein kinase (p38 MAPK) is activated upon S282 dephosphorylation, initiating this apoptotic process.

\section{Results}

\section{S282, but not S279, mutation induced cardiomyocyte apoptosis via Fas/FADD activation in vitro}

Previously, we demonstrated that $\mathrm{Cx} 43$ phosphorylation at S282 (pS282) mediates gap coupling in neonatal rat ciency at serine 282 (S282) activates factor-associated suicide 


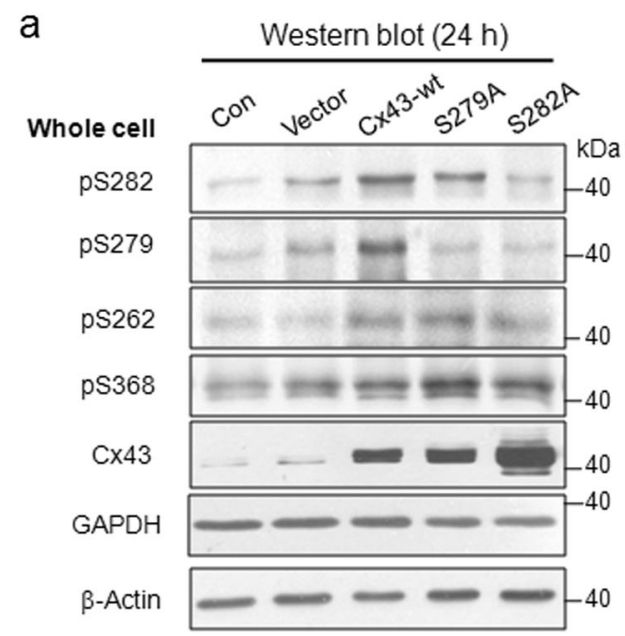

b
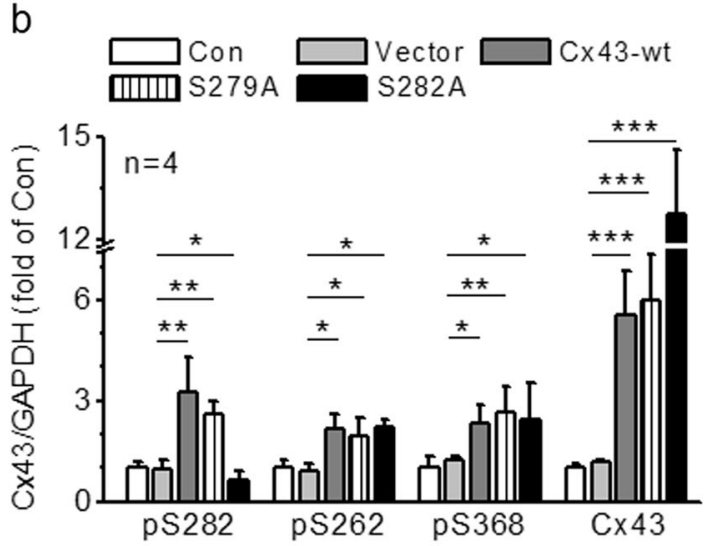

C

d

\section{Junctional fraction}

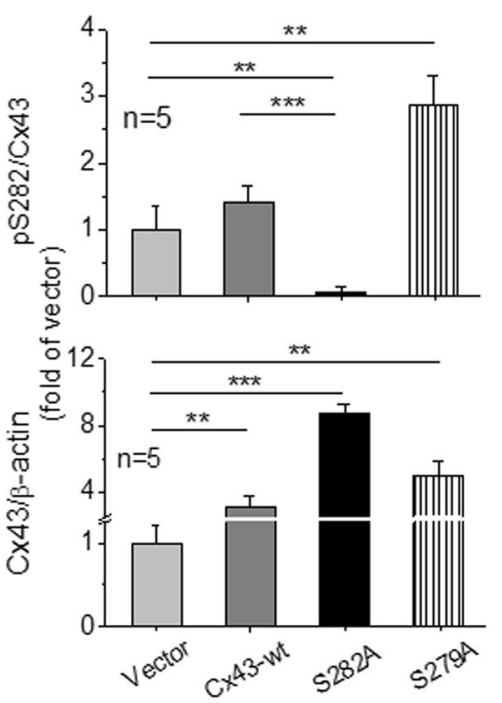

Non-junctional fraction

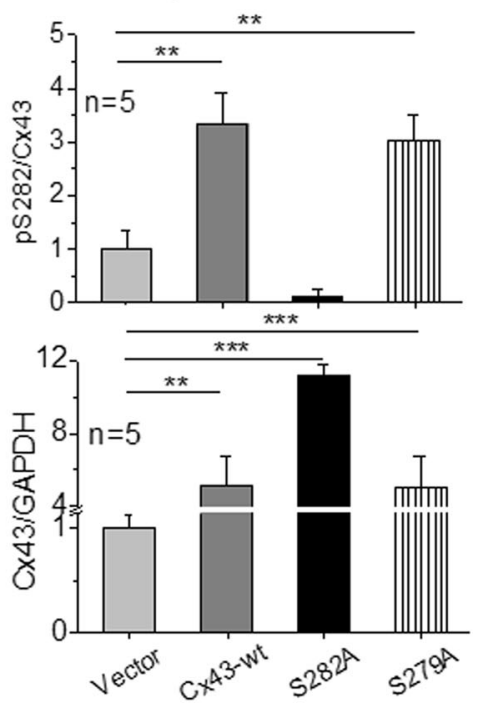

Fig. 2 Cx43 expression and distribution in NRVMs transfected with different mutants. a-d NRVMs were transfected with virus carrying Cx43-wt, $S 279 A$ or $S 282 A$ gene (20 m.o.i. for each) for $24 \mathrm{~h}$ and lysed by RIPA $(\mathbf{a}, \mathbf{b})$ or RIPA + junctional/non-junctional isolation treatment $(\mathbf{c}, \mathbf{d})$. The lysates were analyzed by Western blot (a, c), and the fold changes in phosphorylated S282 (pS282), pS262, pS368 and Cx43 abundances in relative to those of vector cells (after normalized with GAPDH or $\beta$-actin) were thereby detected $(\mathbf{b}, \mathbf{d})$. Junctional and non-junctional fractions were referred to cell-membrane and cytosolic fractions to detect $\mathrm{Cx} 43$ abundance distributed in both regions (see Methods), respectively, and no difference in their pattern between the two regions was found among the different treatments. $* P<0.05$, $* * P$ $<0.01, * * * P<0.001$, unpaired two-tailed Student's $t$ test, $n=4-5$ independent experiments for each group as indicated ventricular myocytes (NRVMs) by transfecting an adenovirus carrying S282 mutant gene substituted with alanine (S282A) for $24 \mathrm{~h}$ [17]. Unexpectedly, after further culturing, S282A-transfected NRVMs continued to die, while cells transfected with the wild-type $C x 43$ gene (Cx43-wt) or the mutant S279A remained intact (Fig. 1a, b and Videos 1, 2). Using flow cytometry to detect annexin V-FITC/PI staining, S282A cells displayed dramatic apoptosis response after transfected for $36 \mathrm{~h}$ (Supplementary Fig. 1). In addition, S282A cells showed uncoupled $\mathrm{Ca}^{2+}$ signaling and inhibited Lucifer Yellow (LY) uptake, suggesting a blockaded intercellular coupling (Fig. 1c-e). Western blot analysis displayed reduced $\mathrm{pS} 282$ and high $\mathrm{Cx} 43$ overexpression that was distributed similarly to $\mathrm{Cx} 43$ in the membranes of S279A and Cx43-wt cells (Fig. 2a-d), which was also evidenced via immunostaining (Fig. 1c). Phosphorylation levels at other C-terminal serine sites, including S262 and S368, were unaffected by S282 or S279 mutation (Fig. 2a, b).

To investigate the apoptosis pathway responsible for NRVM death, cells after transfection for $28 \mathrm{~h}$ were used and detected by annexin V-FITC/PI staining as well as an ELISA assay kit. We found a three-fold increase in 

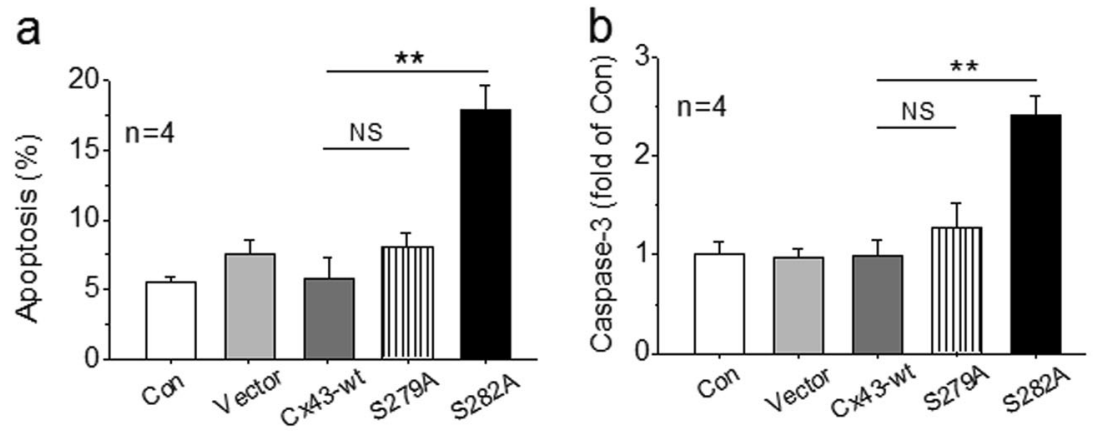

C
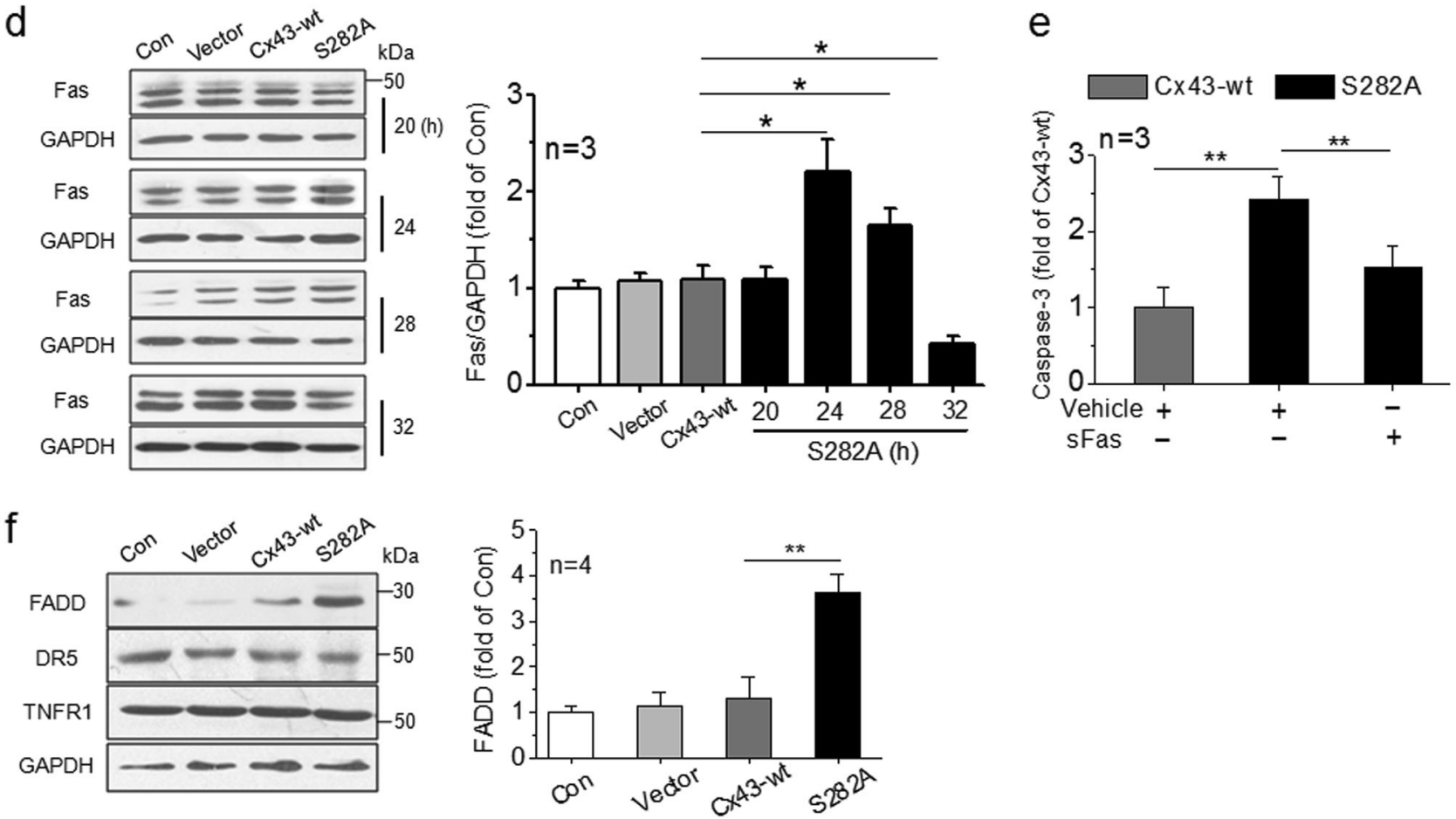

Fig. 3 Expression of S282 mutant with alanine activated Fas-apoptotic pathway. a, b Apoptosis in NRVMs transfected with virus carrying Cx43-wt, $S 279 A$ or $S 282 A$ gene for 28 h (20 m.o.i. for each), respectively, was detected by annexin V-FITC/PI assay using flow cytometry (a) and caspase- 3 ELISA kit (b), $n=4$ independent determinations for each group. c Caspase- 8 activity in these transfected NRVMs was determined by Western blot with a specific antibody against caspase-8, $n=3$ independent determinations for each group. d, e Transfectiontime-dependent activation of Fas in NRVMs with S282A mutant was analyzed by Western blot, and fold changes of Fas in these cells after normalized with GAPDH, respectively, in relative to those of control

cells were thereby detected (d). The activation of caspase- 3 by $\mathrm{S} 282 \mathrm{~A}$ was significantly inhibited by soluble Fas (sFas, $3 \mu \mathrm{g} / 60-\mathrm{mm}$ dish, added to the dish when virus was added) (e), $n=3$ independent determinations for each group. $\mathbf{f}$ The levels of FADD, DR5, and TNFR were determined by Western blot in cells transfected with S282A or $\mathrm{Cx} 43$-wt for $28 \mathrm{~h}$ and the fold increases in their abundances in relative to those of control (after normalized with GAPDH) were thereby detected, $n=3-4$ independent determinations for each group as indicated. $* P<0.05$, $* * P<0.01$, NS: not significant, unpaired two-tailed Student's $t$ test or one-way ANOVA (e)

apoptosis-positive cells and a two-fold increase in caspase-3 activity in S282A-transfected cells compared with Cx43-wt cells (Fig. 3a, b). Because both caspase- 9 activity and the release of cytochrome $\mathrm{C}$ from the mitochondria, the indication of mitochondrial apoptotic activation, did not differ between S282A and Cx43-wt-transfected NRVMs (Supplementary Fig. 2), we explored the Fas/FADD/caspase-8 apoptotic pathway and found markedly increased cleaved caspase-8 and Fas expression in S282A cells (Fig. 3c, d). The inhibition of caspase- 3 activation by soluble Fas ( $\mathrm{sFas}$ ), a blocker of Fas activation [18], further supported the Fas activation involved in S282A-mediated cell death (Fig. 3e). In addition, Fas was activated in a transfection-timedependent manner, accompanied by a marked activation of FADD but no other apoptotic proteins such as death receptor 5 (DR5) or tumor necrosis factor receptor (TNFR) (Fig. 3f). Therefore, these data suggest that deficient $\mathrm{Cx} 43$ phosphorylation at S282, but not at S279, causes cardiomyocyte apoptosis via the activation of Fas/FADD/caspase8 pathway. 

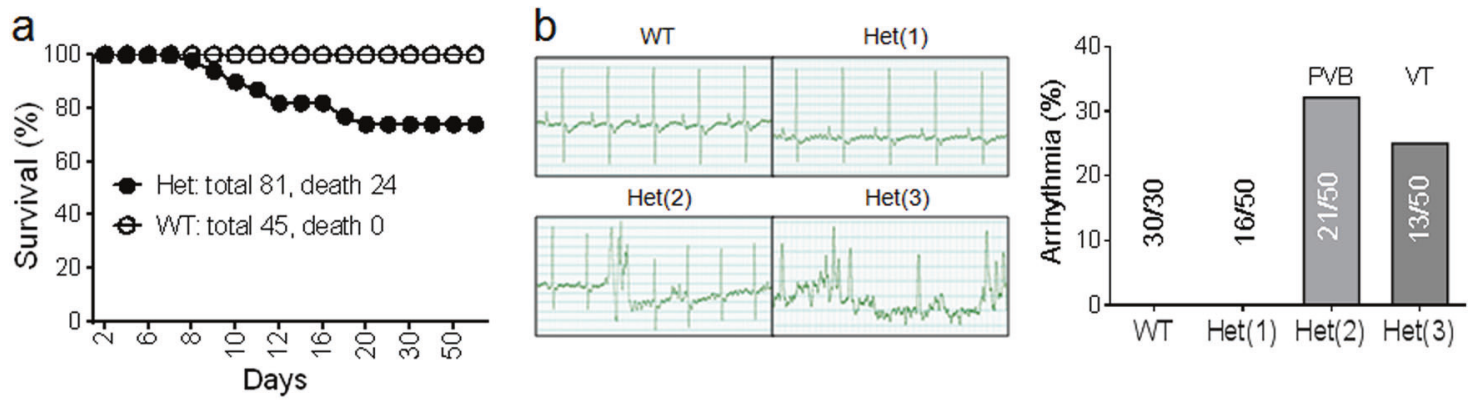

C

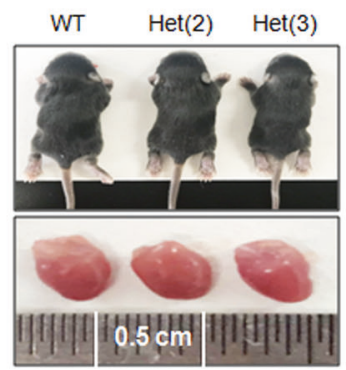

d
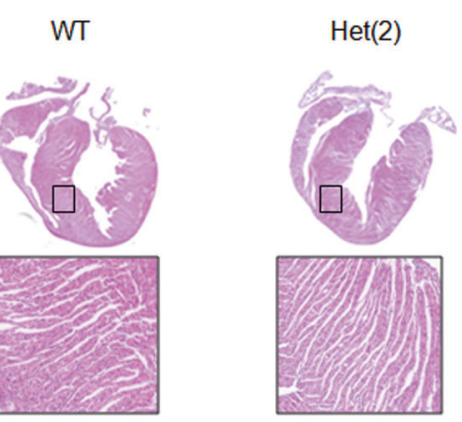

Het(3)
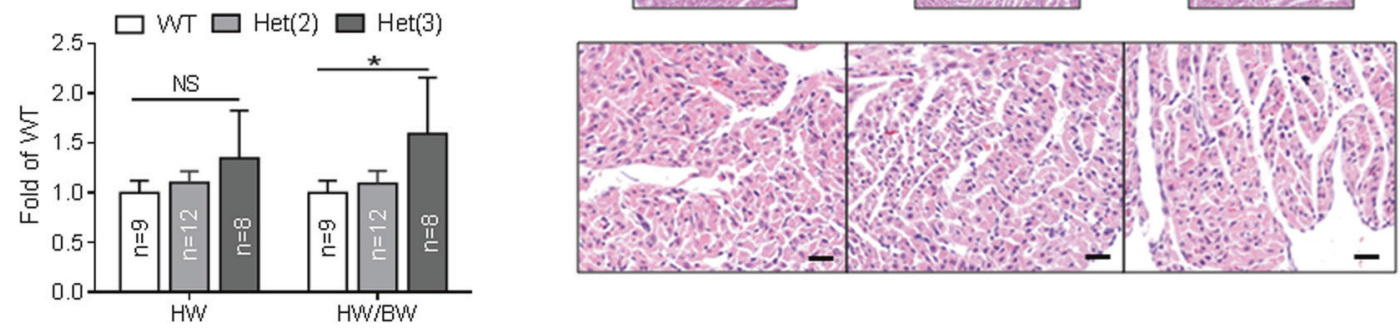

Fig. $4 \mathrm{Cx} 43 \mathrm{~S}^{2} 82 \mathrm{~A}^{+/-}$mice exhibited arrhythmias and cardiomyocyte apoptosis. a Neonatal lethality (24/81) occurred in heterozygous mice (Het) between day 9 and 21 after birth, while no death was found in wild-type mice (WT). b Typical surface ECG recordings show different changes in Het; generally normal ECG, premature ventricular beat (PVB) and ventricular tachycardia (VT), while no arrhythmia was found in WT. Thereby, three groups: Het(1), Het(2) and Het(3) were divided according to the severity of arrhythmias. c Representative photos of animals and hearts from WT, Het(2) and Het(3) groups, and

\section{S282 mutation selectively induced cardiomyocyte apoptosis independent of gap junction blockade}

Because Cx43 site mutations can affect some tissue development [9-12], primary cultured myofibroblasts and vascular smooth muscle cells after three passages were used and transfected with these mutants for $48 \mathrm{~h}$ to detect the S282A proapoptotic effects on the non-cardiomyocytes. Generally, similar changes in pS282 and $\mathrm{Cx} 43$ expressions and LY uptake in cardiomyocytes were observed in both myofibroblasts and vascular smooth muscle cells (Supplementary Fig. 3a-d) and in HEK293 cells [17]. However, S282 mutant did not affect cell shape, growth, and proliferation in all these types of cells.

Previous study showed that S282 dephosphorylation suppressed NRVM intercellular communication (Fig. 1c-e) [17], we treated NRVMs for $24 \mathrm{~h}$ with the gap junction uncoupler, carbenoxolone (CBX) [19], or with aminoethyl

comparisons of the heart weight (HW) and ratio of heart/body weight (BW) between WT and Het groups, $n=8-12$ mice for each group as indicated. d Hearts from different groups stained with H\&E showed no obvious morphological change, except for a noticeable increase in the extracellular space in Het(2) and (3) ventricles. Scale bar: $20 \mu \mathrm{m}, n$ $=5$ animals for each group. All the animals were detected between day 10 and 14 after birth. $* P<0.05$, NS not significant, unpaired twotailed Student's $t$ test

diphenylborinate (2-APB), which blocks the inositol 1,4,5trisphosphate receptor, and inhibits gap communication and Cx43 phosphorylation at $\mathrm{S} 279 / 282$ as well $[17,20]$, to eliminate the possible effect of gap blockade on cell survival. Both compounds hampered the spread of 6-carboxyfluorescein (6-CF) and synchronized $\mathrm{Ca}^{2+}$ oscillations in monolayer NRVMs, thus indicating gap uncoupling. However, 2-APB significantly changed the cell shape, reduced $\mathrm{Cx} 43$ phosphorylation at $\mathrm{S} 282$ and activated Fas/FADD (Supplementary Fig. 4a-e), while CBX did not. This demonstrated that $\mathbf{S} 282$ dephosphorylation rather than gap uncoupling was responsible for activating cell apoptosis. This finding was further supported by the observation that using an adenovirus carrying Cx43-miRNA to knock down $\mathrm{Cx} 43$ expression (by approximately 90\%) decreased LY uptake and coordinated $\mathrm{Ca}^{2+}$ oscillations but did not induce morphological changes or Fas/FADD activation in NRVMs (Supplementary Fig. 5a-d). 
a

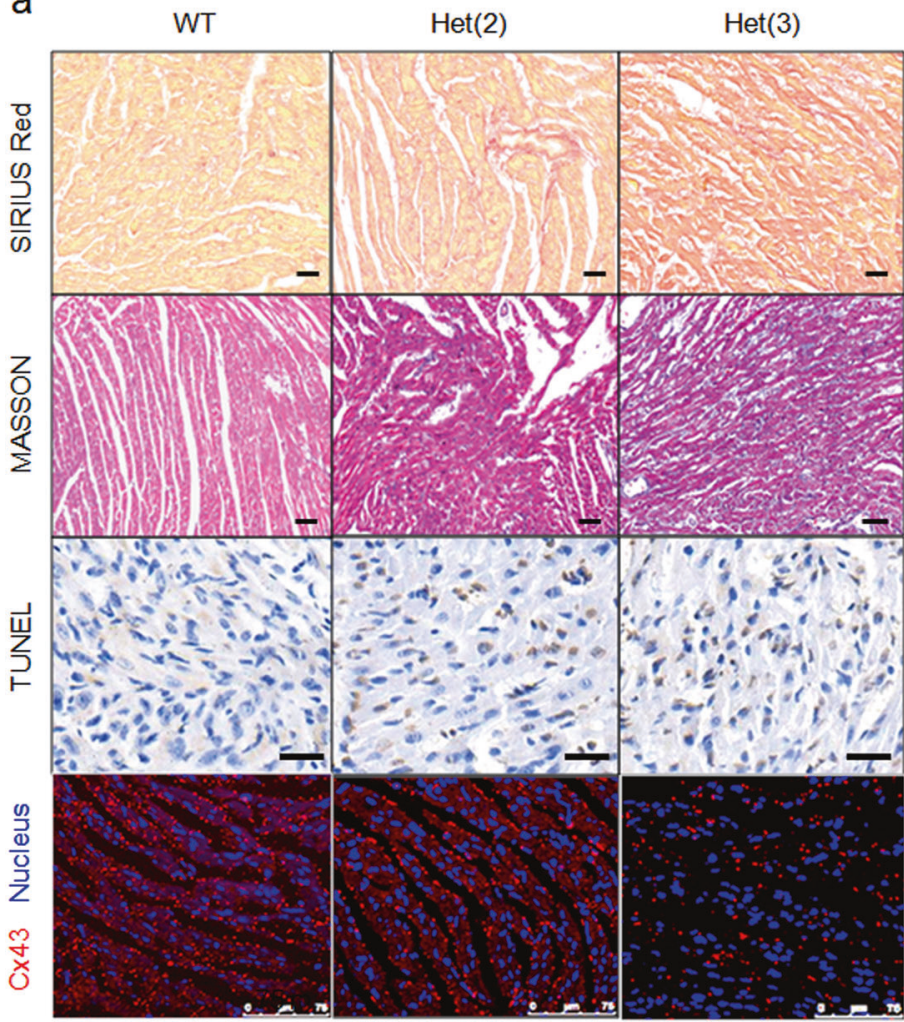

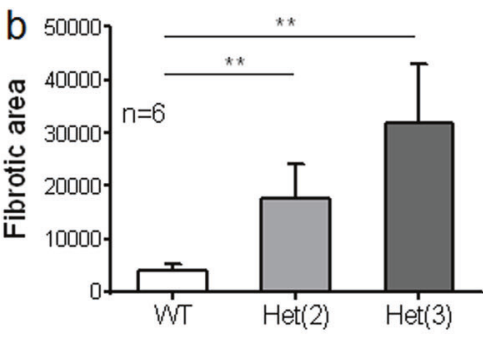
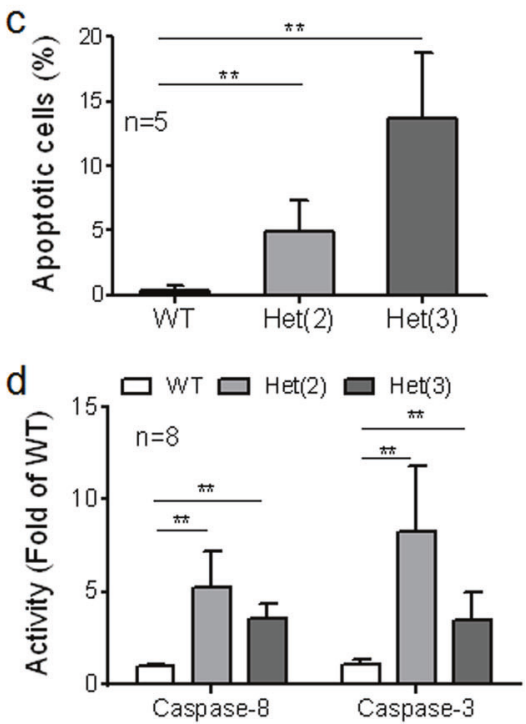

Fig. $5 \mathrm{Cx} 43 \mathrm{~S} 282 \mathrm{~A}^{+/-}$mice exhibited myocardium apoptosis and fibrosis. a-c Mouse ventricles from three groups were stained with antibody against $\mathrm{Cx} 43$ or with SIRIUS Red, MASSON or TUNEL as indicated. Nucleus was stained with Hochest33342. Scale bar: $75 \mu \mathrm{m}$ for $\mathrm{Cx} 43$ staining and $20 \mu \mathrm{m}$ for other images (a). The analysis data for SIRIUS Red-positive signal (b) and TUNEL-positive cells (c) were

\section{Cx43-S282A heterozygous mice displayed cardiomyocyte apoptosis and arrhythmias in vivo}

To further investigate the novel effect of $\mathrm{Cx} 43$ phosphorylation deficiency at $\mathbf{S} 282$ on cardiomyocyte survival and arrhythmogenesis, we established Cx43-S282A mice, generated and identified their offspring, and evaluated their hearts. Their offspring (156 mice) were 36\% wild-type (WT), 64\% heterozygous (Het) and 0\% homozygous (Hom) from Het mating pairs (Supplementary Fig. 6a-d). In addition, $\sim 30 \%$ of the Het mice died between days 9 and 21 after birth (Fig. 4a). Thus, we focused on the Het mice from days 10 to 14 after birth and investigated whether their hearts incurred apoptotic injury.

Neonatal Het mice were measured from day 10 after birth and $66 \%$ Het had differing degrees of spontaneous arrhythmias. These mice were divided into three groups: Het(1) had generally normal ECG; Het(2) had premature ventricular beats (PVB); and Het(3) had ventricular tachycardia (VT) (Fig. 4b). Notably, the Het(3) group's heart/weight $(\mathrm{H} / \mathrm{W})$ ratio was significantly increased, displayed, $n=5-6$ animals for each group as indicated. d Caspase-8/3 activities determined by ELISA kits, respectively, illustrated significant activations of caspase-8/3 in both Het(2) and Het(3) mice, $n=$ 8 mice for each group. Note that Het(3) mice showed severer apoptosis and fibrosis than Het(2) mice. $* * P<0.01$, unpaired two-tailed Student's $t$ test

while their heart development and morphologies appeared normal (Fig. 4c). Accordingly, both Het(2) and Het(3) mouse ventricles exhibited increased extracellular space (Fig. 4d); increased TUNEL-, Masson- and Sirius Redpositive stained cells (indicative of fibrosis; Fig. 5a-c); and caspase-8/3 and Fas/FADD activation (Figs. 5d and $6 \mathrm{a}, \mathrm{d})$. The abundance of pS282 was gradually reduced from the Het(1) to the Het(3) hearts, but $\mathrm{Cx} 43$ expression was only reduced in the $\operatorname{Het}(3)$ hearts with unaltered expression of $\mathrm{N}$-cadherin, the adherens junction protein in the heart (Fig. 6a, c). Importantly, all abnormal alterations were strongly correlated with pS282 level (Fig. 6e) but not $\mathrm{Cx} 43$ or pS368 abundance, both of which were unchanged in the Het(2) hearts compared with the WT hearts (Fig. 6a, c). Additionally, the $\mathrm{Cx} 43$ and pS282 expressions remained unchanged in the bone, brains and livers in the Het(3) mice (Fig. 6b), suggesting that only the heart is susceptible to S282 mutation after alanine substitution.

Similar to that in S282A-treated NRVMs (Supplementary Fig. 2), the mitochondrial apoptotic pathway was not 


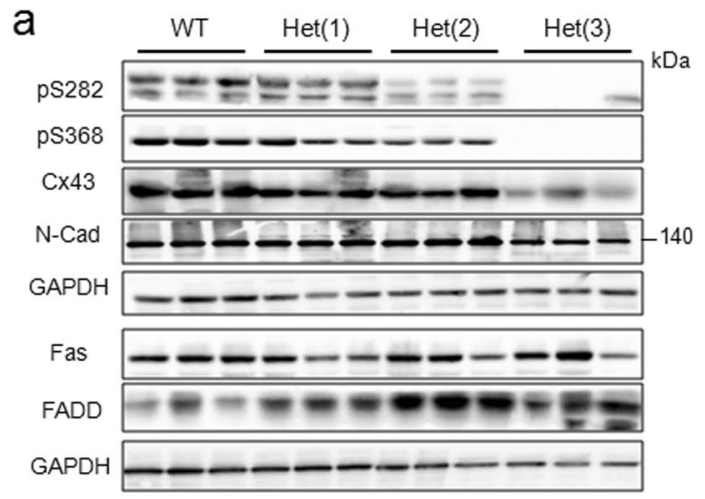

b

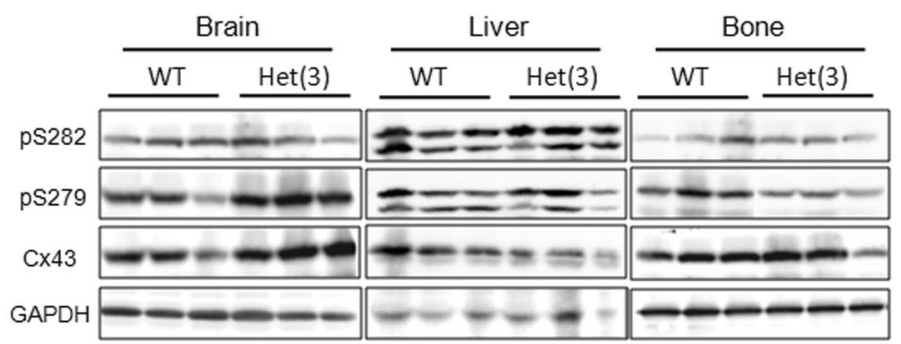

C

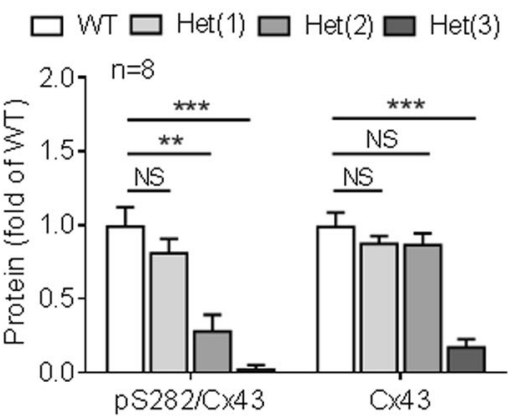

d

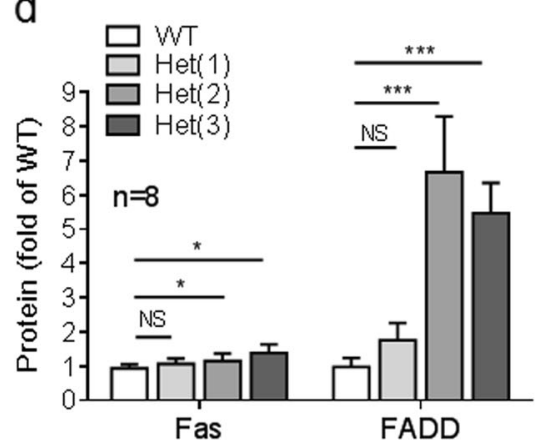

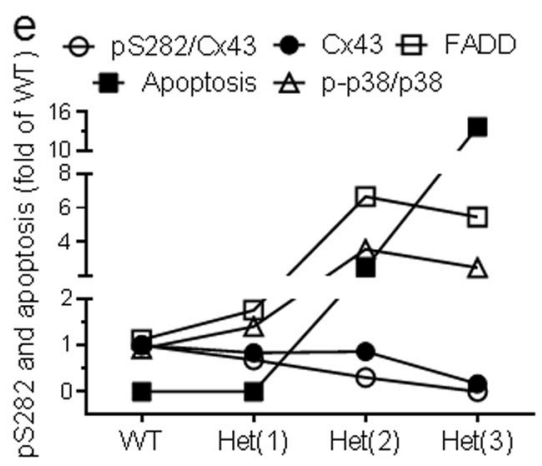

Fig. $6 \mathrm{Cx} 43 \mathrm{~S} 282 \mathrm{~A}^{+/-}$mice exhibited an activation of Fas/FADD pathway. a-d Lysates from ventricles of WT and Het(1)-(3) (a) and from brain, liver and bone tissues of WT and Het(3) mice (b) were analyzed by Western blot with specific antibodies as indicated, respectively, and fold changes in pS282 and Cx43 (c) and Fas/FADD (d) in relative to those in WT ventricles (after normalized with GAPDH) were thereby detected, $n=8$ mice for each group in all the

significantly activated in the Het(2) mice (Supplementary Fig. 7).

\section{p38 MAPK activation caused S282 dephosphorylation-induced cell apoptosis}

Next, we investigated how pS282 deficiency induces cardiomyocyte apoptosis. MAPK, especially p38 MAPK, has been proposed to play an important role in inducing myocardial damage [21-23], and Fas/FADD activation appears to be involved in its signal transduction [24, 25]. Therefore, we examined the abundances of phospho-p38 (Thr180/ Tyr182) and p38 MAPK $\alpha$, the dominant active isoform during myocardial ischemia [21, 22], using specific antibodies, and found that p-p38 abundance was significantly elevated relative to p38 MAPK in S282A NRVMs and in the S282A Het hearts (Fig. 7a-d) compared with those of the counterpart controls. Twenty hours before Fas activation (Fig. 3d), p-p38 MAPK was significantly higher in S282A NRVMs than in Cx43-wt cells and reached its highest level $24 \mathrm{~h}$ after viral infection (Fig. 7e). Inhibiting p38 MAPK by SB203580 $(10 \mu \mathrm{M})$ significantly prevented S282A-induced panels, except for five mice for each group in b. e Comparing the values of pS282, Cx43, FADD, TUNEL positive cells (from Fig. 5c) and p-p38/p38 MAPK (from Fig. 7b, d) among the ventricles from different groups showed consistence in the activation of apoptosis pathway (p38/FADD), which was correlated with the level of pS282 but not $\mathrm{Cx} 43$ expression. $* * P<0.01$, $* * * P<0.001$, NS not significant, unpaired two-tailed Student's $t$ test

activation of p38 MAPK and Fas, and decreased the cell viability (Fig. 7f-h).

In addition, double immunofluorescence staining with specific antibodies for Cx43 (red) and p38 MAPK (green) displayed markedly increased colocalization along with Cx43 distribution in S282A-transfected NRVMs compared with $\mathrm{Cx} 43$-wt cells (Fig. 8a, upper panels). Compared with S282A-negative cells (yellow arrows) and Cx43-wt cells, Cx43/p38 were colocalized throughout the entire transfected cell, predominantly in the cell membrane and lateral side of the nucleus (white arrows), represented by Pearson's correlation coefficient (see Methods, Fig. 8b). More colocalized Cx43/p38 dots were found in neonatal Het(2) ventricles than were found in the WT ventricles (Fig. 8a low panels). Physical interactions between $\mathrm{Cx} 43$, p38 and FADD were detected by coimmunoprecipitation using a specific antibody for $\mathrm{Cx} 43$ to precipitate p38 and FADD. More abundant p-p38, p38 and FADD were observed in the Het(2) ventricles than in the WT hearts (Fig. 8c). Similarly, enhanced p38 phosphorylation and physical interaction of p38 and Cx43 were also found in S282A-transfected cardiomyocytes (Fig. 8d). 

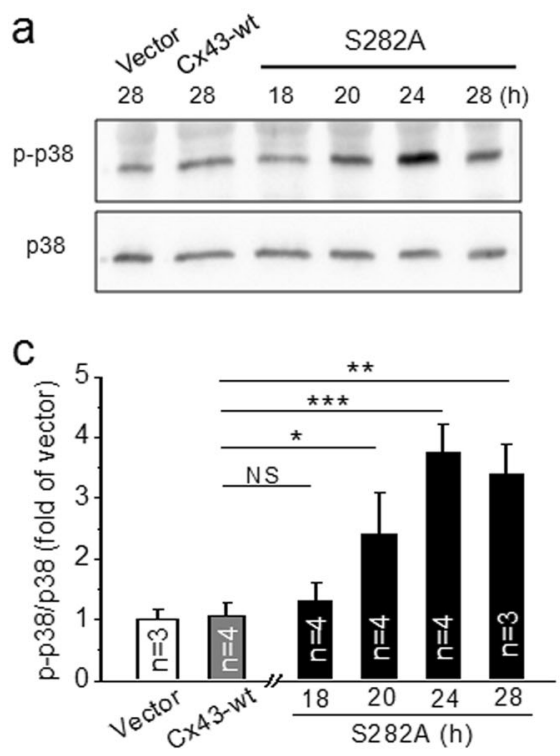

f

Cx43-wt S282A SB+S282A

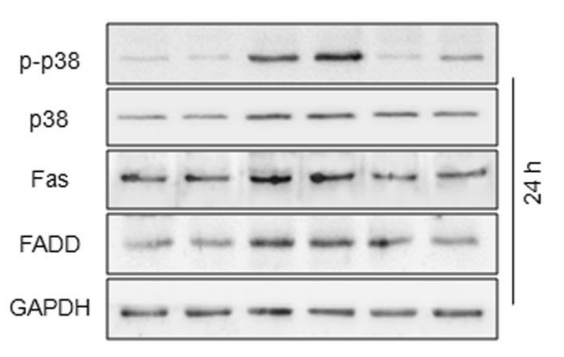

b
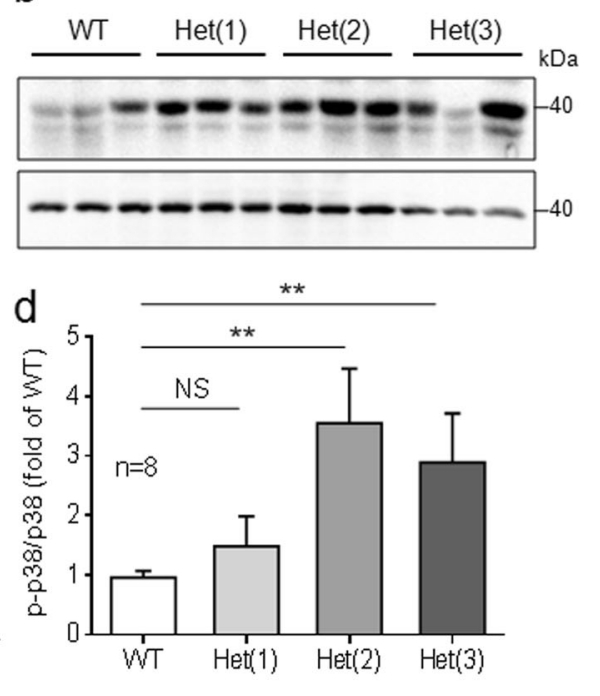

g

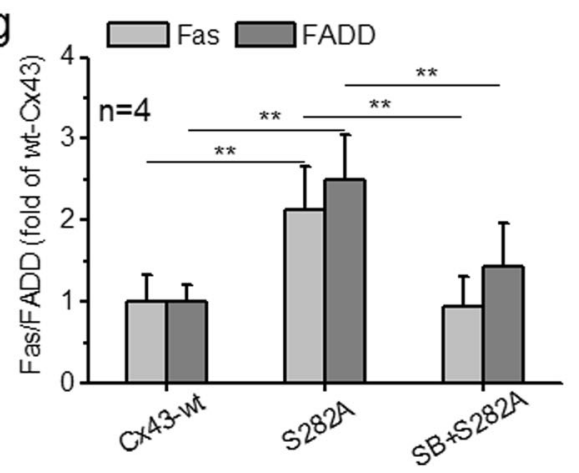

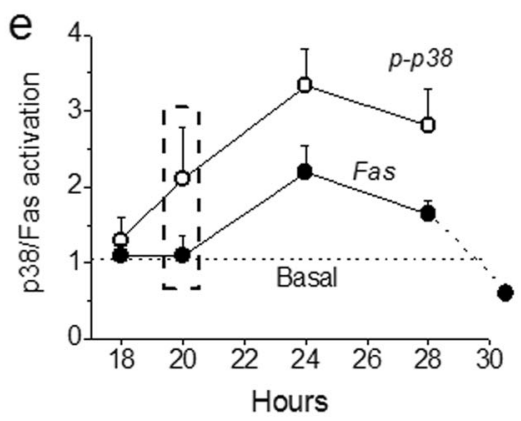

h
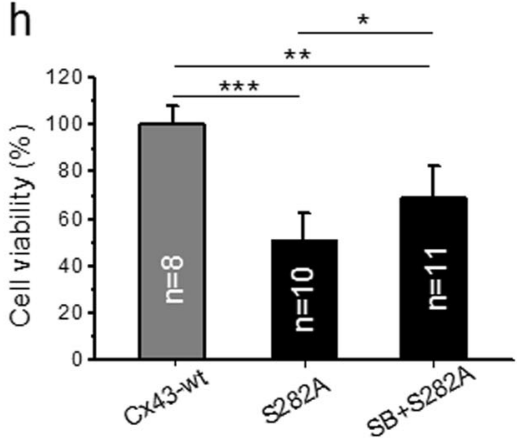

Fig. 7 Activation of p38 MAPK responsible for the cell death in S282 mutated NRVMs and Cx43-S282A ${ }^{+/-}$mice. a-d Lysates from S282A-transfected NRVMs (a) and ventricles of WT and Het mice (b) were analyzed by Western blot using specific antibody for phosphorylated p38 MAPK (p-p38) or p38 MAPK, and fold increases in pp38 in cells (c) and ventricles (d) after normalized with p38 MAPK in relative to their counterpart controls were thereby detected, $n=3-4$ independent determinations (c) and 8 mice (d) for each group as indicated. e The activation of p38 MAPK was transfection-time- dependent (data from a) and compared with that in Fas activation (data from Fig. 3d) in S282A transfected cells. $\mathbf{f}-\mathbf{h}$ The inhibitory effect of SB203580 (SB, $10 \mu \mathrm{M}$, added at the time of transfection and harvested $28 \mathrm{~h}$ after) on Fas/FADD activation (f, $\mathbf{g}$ ) and the lowered cell viability (h) induced by S282A transfection were detected by Western blot and 3-(4,5-Dimethylthiazol-2-yl)-2,5-diphenyltetrazolium bromide (MTT) method, respectively, $n=4$ (g) and 8-11 (h) independent determinations for each group as indicated. $* P<0.05, * * P<0.01$, $* * * P<0.001$, unpaired two-tailed Student's $t$ test $(\mathbf{c}, \mathbf{d})$ and one-way ANOVA $(\mathbf{g}, \mathbf{h})$

\section{S282 phosphorylation deficiency and p38 MAPK/ Fas/FADD pathway activation in doxorubicin- induced myocardial injury}

Finally, we tested whether S282 dephosphorylationassociated apoptosis occurred in doxorubicin (Dox)induced myocardial injury because this chemotherapy drug is prone to inducing myocardial apoptosis, cardiomyopathy and heart failure [26, 27]. Mice treated with Dox for 5 days displayed disarranged myocardium with increased extracellular space, matrix collagen expression, and myocyte apoptosis (Supplementary Fig. 8). Notably, Cx43 lateralization, reduced pS282 level, and Fas/FADD and caspase$8 / 3$ activations were all found in the Dox-treated mouse ventricles, suggesting that S282 deficiency is involved in Dox-induced heart lesions.

\section{Discussion}

As more connexin-interacting proteins are found, $\mathrm{Cx} 43$ gap junctions act not only as channels between neighboring cells, but also as modulators regulating cell function, survival and death via mostly undefined mechanisms [13, 14, 28, 29]. Here, we demonstrated that Cx43-phospho-S282 plays essential roles in regulating cardiomyocyte survival and electrical stability. S282 phosphorylation deficiency by genetic or chemical approaches triggered cardiomyocyte 

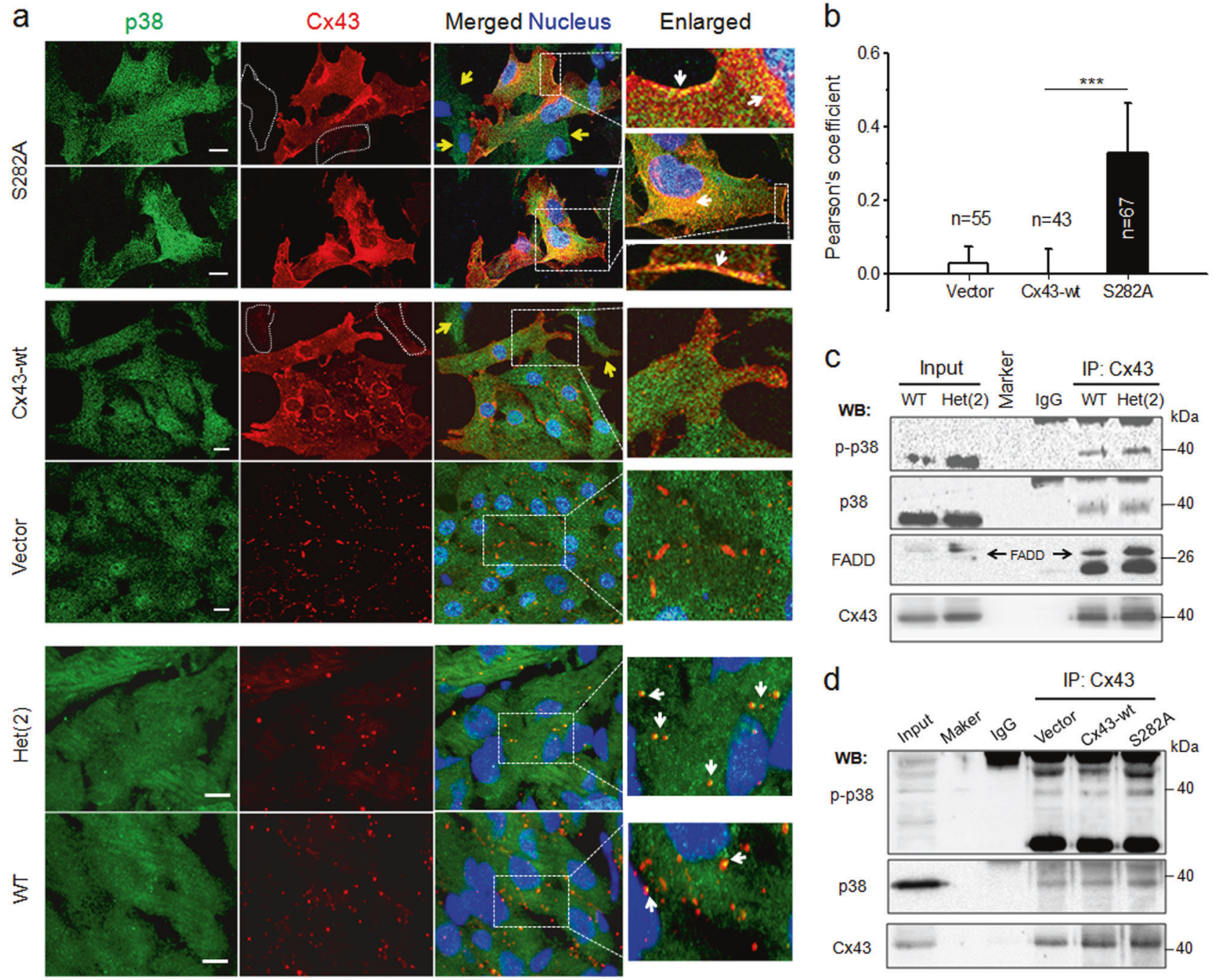

Fig. 8 Enhanced physical interaction between p38 MAPK and $\mathrm{Cx} 43$ in S282A transfected cardiomyocytes and Cx43 S282A ${ }^{+/-}$hearts. a Representative images of NRVMs transfected with adenovirus and ventricles from WT and Het(2) mice, as indicated, and double-stained with antibodies specific for p38 MAPK (green) and Cx43 (red). The cells indicated with dot irregular shapes and yellow arrows were S282A-negative cells. White arrows point to p38 MAPK colocalized with $\mathrm{Cx} 43$ in S282A-positive cell surface and lateral side of the nucleus, and in ventricles of WT and Het(2) mice in enlarged images. Nucleus was stained with Hochest33342, scale bar: $10 \mu \mathrm{m}, n=3$ independent experiments for cell experiment and four hearts for

death and disordered electrical transients in vitro under basal conditions (Fig. 1, and Supplementary Figs. 1 and 4), which could not be rescued by Cx43-wt or Cx43-S282D gene coexpression (substituted with aspartate as phospho-mimicking, data not shown), indicating that S282A exhibited a strong dominant negative effect. Moreover, S282A-homozygous mice ceased to develop at the embryonic stage (Supplementary Fig. 6) as reported in other $\mathrm{Cx} 43$ residue-mutated mice $[9,10]$. Heterozygous mice could develop, but more than $60 \%$ revealed spontaneous ventricular arrhythmias from the neonatal stage, and some died before maturation (Fig. 4), in

ventricle test in each group. b The quantitative analysis of $\mathrm{p} 38 / \mathrm{Cx} 43$ colocalization, represented by Pearson's correlation coefficient (see Methods), in adenovirus transfected-NRVMs, $n=43-67$ cells as indicated, $* * * P<0.001$, unpaired two-tailed Student's $t$ test. c Ventricle lysates from WT and Het(2) mice were immunoprecipitated with anti-Cx43 antibody and then Western blotted with anti-p-p38, anti-p38 or anti-FADD antibody, $n=3$ independent experiments from 32 mice for each group. d Lysates from vector and Cx43-wt or S282Atransfected cardiomyocytes were immunoprecipitated with anti-Cx43 antibody and then Western blotted with anti-p-p38 or anti-p38 antibody, $n=3$ independent experiments for each group

consistent with the findings in Cx43 knockout or site-mutated mice [7-10]. Importantly, these heterozygous mice exhibited a novel phenotype - cardiomyocyte apoptosis and myocardial fibrosis (Figs. 5-7)—while other tissues, including bone, brain and liver, developed normally with unchanged S282 phosphorylation and Cx43 expressions. This is unlike the phenotype in I130T mice that exhibited abnormal bone development and arrhythmias without cardiac injury [9]. In addition, primary cultured rat myofibroblasts and vascular smooth muscle cells retained normal growth and differentiation after S282 phosphorylation was blocked (Supplementary 
Fig. 3). Thus, these results demonstrated that only cardiomyocytes occur death upon Cx43-S282 dephosphorylation.

Activation of the apoptotic pathway by deficient S282 phosphorylation was likely unassociated with mitochondrial activation (intrinsic apoptotic pathway) (Supplementary Figs. 2, 7), while activation of the Fas/FADD/caspase- 8 pathway (extrinsic apoptotic pathway) mechanistically should account for the deficient pS282-induced apoptosis (Figs. 3, 6 and Supplementary Fig. 4), which is also activated in severe cardiac diseases. Here, p38 MAPK (a critical stress kinase in the heart) appeared to be the upstream of Fas/FADD activation and initiated S282A-induced cardiomyocyte apoptosis because S282A induced a transfection-time-dependent activation of p38 MAPK before activating Fas/FADD. Additionally, inhibiting endogenous p38 MAPK activity with SB203580 blocked S282A-induced Fas activation and cell death (Fig. 7). Further, the physical interaction between $\mathrm{Cx} 43$, p38 and FADD, and p38 phosphorylation were enhanced in S282mutated NRVMs and heterozygous hearts (Fig. 8). It has already been found that p38 MAPK interacts with $\mathrm{Cx} 43$ in the heart [30-32], and activation of Fas/caspase-3 is likely involved in p38-induced cardiomyocyte death [24, 25]. p38 MAPK overexpression leads to a proapoptotic effect on cardiomyocytes, whereas inhibiting p38 MAPK activity alleviates cardiac infarction and helps preserve cardiac function following harmful stress [23, 24, 33]. Therefore, this study provided a novel linkage between $\mathrm{Cx} 43$-regulated cell survival and p38 MAPK-mediated apoptosis, which may explain how $\mathrm{Cx} 43$ remodeling is associated with cardiomyocyte apoptosis and participates in myocardial injury in several severe heart diseases [4-6, 14-16, 29].

Targeting p38 MAPK has been suggested as a promising direction for drug development against cardiac injury. However, noticeable cardiac toxicity due to p38 MAPK inhibitors suppressing the benefits of endogenous p38 MAPK activity hinders its application [21, 22]. Recently, some noncanonical MAPK kinase-independent activation mechanisms were identified [34-36]. Specifically, p38 MAPK autophosphorylation via binding to TGF- $\beta$-activated protein kinase 1-binding protein is the mechanism that activates p38 MAPK during myocardial ischemia. Therefore, this provides a specific target and new strategy to prevent p38 MAPK activation during myocardial injury while maintaining its benefits [36]. The association between deficient Cx43 phosphorylation at S282 and p38 MAPK activation found in this study may also provide new insights into understanding $\mathrm{Cx} 43$-mediated cardiomyocyte death and additional p38 activation pathways.

Cx43 mutations or complete knockout can induce various arrhythmias and developmental problems in animals and humans, in which severe ventricular arrhythmias have been recognized as a cause of death [7-12]. In addition to ventricular arrhythmias, we found that dispersed cardiomyocyte apoptosis together with p38/Fas/FADD activation and cardiac fibrosis were highly correlated with the degree of S282 dephosphorylation in S282 heterozygous mice, suggesting that cardiomyocyte homeostasis and electrical stability depend heavily on S282 phosphorylation. In addition, Cx43 expression in the heart was also hampered by S282 dephosphorylation in het(3) mice. Nevertheless, this is unlikely to induce cardiomyocyte apoptosis because het(2) mice showed myocardial apoptosis and fibrosis with unaltered Cx43 expression and distribution (Figs. 5, 6). Moreover, because Cx43-deficient NRVMs retained their normal shape and growth (Supplementary Fig. 5), and Cx43-knockout mice developed to adulthood with unaltered cardiac structure and function except for occasional severe ventricular arrhythmias [7-9, 12], Cx43 expression abundance change is unlikely responsible for S282A-induced apoptosis.

Recent evidence shows that $\mathrm{Cx} 43$ is involved in regulating tissue and organ development and cell growth, proliferation, homeostasis and death [13, 14, 28]. Under severe pathological conditions, including cardiac ischemia, failure and arrhythmia, Cx43 undergoes remodeling, including downregulated $\mathrm{Cx} 43$ expression and C-terminal serine dephosphorylations, such as S262 [29], S365 [37], S368 [29], S297 [38], and S325/328/330 [15, 39]. This pathological dephosphorylation modification greatly impairs cardiac electrical and pump stabilities, and even enhances myocardial injury via undefined mechanisms [13-15]. In this study, S282 dephosphorylation induced cardiomyocyte apoptosis under normal conditions. Moreover, significant phospho-S282 downregulation occurred in both doxorubicin-injured heart (Supplementary Fig. 8) and ischemic/reperfusion heart (described in another manuscript), reflecting a potential mechanism underlying the cardiomyocyte apoptosis that occurred in these pathologies.

In summary, this study demonstrated that $\mathrm{Cx} 43$ phosphorylation at S282 mediates cardiomyocyte survival and electrical stability. Deficiency in this residual phosphorylation triggers cardiomyocyte death by activating p38 MAPK and the Fas/FADD pathway. This study may suggest a previously unknown mechanism for Cx43-mediated cardiomyocyte survival and apoptosis under physiological and pathophysiological conditions.

\section{Materials and methods}

The detection of cell death by fluorescent dye YOYO-1 iodide using IncuCyte ZOOM (Essen Bioscience), isolations of vascular smooth muscle cells and cardiomyocyte mitochondrial fraction, the detection of gap exchange by 6 carboxyfluorescein (6-CF) dye transfer analysis and the 
preparation of doxorubicin-induced cardiac injury were described in the online supplementary materials.

Animals were maintained at the Center for Experimental Animals at Capital Medical University (Beijing, China) and maintained on a $12 \mathrm{~h}$ light-dark cycle in a temperaturecontrolled room with access to food and water ad libitum. The animal experiments in this study were approved by the Capital Medical University Animal Care and Use Committee (AEEI-2015-193) and conducted in accordance with the "Guide for the Care and Use of Laboratory Animals" adopted by the Beijing Municipal People's Government.

\section{Generation of Gja1 S282A mutant mice}

S282A-mutant mice were generated at Cyagen Biosciences (Santa Clara, CA, USA) using C57BL/6N embryonic stem cells by standard gene targeting techniques. Briefly, to engineer the targeting vector, the $5^{\prime}$ and $3^{\prime}$ homology arms were amplified from BAC clone RP24-368N3/RP24-289B3 from the C57BL/6J library as a template (Supplementary Fig. 6a). The targeting vector was constructed by sitedirected mutagenesis to replace serine (Ser) with alanine (Ala) at position 282 (S282A single nucleotide polymorphism [SNP] at exon 2) and was transfected into C57BL/6N embryonic stem cells. The targeting vector had a Neo selection cassette flanked by LoxP sites for antibiotic selection as well as diphtheria toxin A (DTA), which was used for negative selection to identify cells carrying the S282A SNP. Embryonic stem cells were injected into mouse blastocysts and transplanted into the uteri of Creexpressing C57BL/6 mice to produce chimeric knock-in (KI) mice and to remove the Neo cassette. After selection, allele presence was verified by Southern blot analysis and DNA sequencing of the PCR products generated using the primers: GGCTGGTAAGGGATATTTGCCTG forward and TCCAAGGGTCTGTATG-CCTCTAAG reverse. Sequence analysis of F0 male mice confirmed the mutation of Ser to Ala (Supplementary Fig. 6b). The chimeric heterozygous F1 mice were interbred to generate S282A homozygous mice. The genotyping strategy for the S282Amutant mice was performed by PCR for the tail DNAs with the specific primers, Gja1_F1: AAGGCTGGTTGCTGAAGGTCCGT and Gja1_R1: TCCCAAAGATGAAGT TGACTCGG.

\section{Isolation and culture of myocytes and transfection with adenovirus}

NRVMs were isolated from 2-day-old Sprague-Dawley rats by enzymatic digestion with $0.1 \%$ trypsin and $0.03 \%$ collagenase as described previously [40]. The isolated cells at the density of $1 \times 10^{6} / \mathrm{ml}$ were cultured for 24,36 or $72 \mathrm{~h}$ as needed and used for $\mathrm{Ca}^{2+}$ transients, LY uptake and cytoimmunostaining, respectively. Cells at the density of $3 \times 10^{6} / \mathrm{ml}$ were used for Western-blot, flow cytometry and 6-CF dye transfer analysis. The cells exhibited $>95 \%$ positive staining for $\alpha$-actinin in this study. Recombinant plasmids and adenoviral vectors carrying wild-type $\mathrm{Cx} 43$ (Cx43-wt), Cx43-S279 substituted with alanine (S279A) and S282 substituted with alanine (S282A) genes were constructed as described previously [17].

The monolayer NRVMs were cultured for $24 \mathrm{~h}$, and transfected with the adenovirus (20 m.o.i. for each), respectively, and cultured further for 24-72 h. For intervention of p38 MAPK or Fas activation, $10 \mu \mathrm{M}$ SB203580 (Selleck) or soluble factor-associated suicide (Fas, $3 \mu \mathrm{g} /$ 60-mm dish) was added to the S282A cells at the same time with virus addition and remained until harvesting.

\section{Assessment of apoptosis}

Myocardial apoptosis was detected by TUNEL kit (Roche Applied Science), caspase-8, -9 and -3 activity by ELISA kits (Invitrogen), and Fas/FADD expression by Western blots. Apoptotic death in cultured cardiomyocytes was also detected by an Annexin V-FITC and propidium iodide (PI) apoptosis kit (Bio Red Biosciences) using flow cytometry.

TUNEL staining of tissue sections was carried out according to the manufacturer's instructions. Briefly, the formalin-fixed paraffinembedded tissue sections were incubated with $20 \mu \mathrm{g}$ proteinase $\mathrm{K}$ for $15 \mathrm{~min}$, and washed several times with PBS. The sections were incubated with TdT-enzyme TUNEL reaction mixture at $37^{\circ} \mathrm{C}$ for $1 \mathrm{~h}$, and then conjugated with horseradish peroxidase dropped on the slides. The 3,3-diaminobenzidine was used as the substrate. Samples were visualized under a Leica inverted microscope at $400 \times$ magnification. The number of apoptotic cardiomyocytes (TUNEL-positive) in relation to the total number of myocytes were calculated from 6 fields in each section.

For flow cytometry, according to the manufacturer's instruction, NRVMs were washed twice with PBS and resuspended in $1 \times$ Binding Buffer at a concentration of $1 \times$ $10^{6}$ cells $/ \mathrm{ml}$. The cell solution $\left(100 \mu \mathrm{l}, 1 \times 10^{5}\right.$ cells $)$ was transferred to a culture tube, and $5 \mu \mathrm{l}$ FITC Annexin V/5 $\mu \mathrm{l}$ PI were added and incubated for $15 \mathrm{~min}$ at $25^{\circ} \mathrm{C}$ in dark. In the end, $400 \mu$ of $1 \times$ Binding Buffer was added to each tube, and cells were analyzed with a BD LSRFortessa, and data were analyzed using BD FACSDiva 7.0 (BectonDickinson).

\section{Histological analyses and immunostaining}

After excision, S282A heterozygous ventricles were fixed in $4 \%$ paraformaldehyde ( $\mathrm{pH} 7.4$ ) for $24 \mathrm{~h}$. Then the samples were transferred into $30 \%$ sucrose solution. Paraffin embedding of paraformaldehyde-fixed tissues were 
sectioned at 3- $\mu$ m thickness. H\&E, SIRIUS Red (Servicebio) and MASSON (Servicebio) staining were carried out according to standard histopathological procedures. Tissue samples were also cryosectioned $(10 \mu \mathrm{m})$ for immunostaining and permeabilized with $0.1 \%$ Triton X-100 for 30 min and blocked with 5\% BSA for $1 \mathrm{~h}$. Antibodies against p38 (rabbit source, CST), Cx43 (goat source, Acris) and $\mathrm{Cx} 43$ (rabbit source, Santa Cruz) were incubated overnight at $4{ }^{\circ} \mathrm{C}$ at the ratio of 1:200. The secondary antibodies, Alexa Fluor 488-labeled donkey anti-rabbit or Alexa Fluor 594-labeled chicken anti-goat (Molecular Probes) were used at a dilution of 1:750 or 1:500, respectively, for $1 \mathrm{~h}$ at room temperature. The nucleus was labeled with Hoechst33258 at the final concentration of $1.0 \mu \mathrm{g} / \mathrm{ml}$ in tissue and cell samples. Fluorescent detection was performed directly on a laser-scanning confocal microscopy (Leica SP8) equipped with a $63 \times$ oil immersion objective NA 1.4. All negative controls were performed using host serum as the primary antibody. For measurement of p38 colocalization with Cx43 in NRVMs, a 3-D z-stack was obtained at $0.5 \mu \mathrm{m}$ intervals and pinhole $=1.0 \mathrm{AU}(1 \mathrm{AU}=95.5 \mu \mathrm{m})$ after mounting. The Pearson's correlation coefficient was calculated for the degree of overlap between fluorescence signals obtained in two channels using Imaris Bitplane software in 3-D whole cell images after background normalization; this value represents the degree of colocalization as strong (0.49-1.0), moderate $(0.1-0.48)$ and weak ( -1 to 0.09$)$ based on previous description [41, 42]. Calculating the colocalization in ventricles was failed due to few positive-signal for calculation.

\section{$\mathrm{Ca}^{2+}$ transient imaging and Lucifer Yellow uptake assay}

Cultured NRVMs were loaded with $4 \mu \mathrm{M}$ Fluo4/AM (Invitrogen) or $2 \% \mathrm{LY}$ (Invitrogen) at $37^{\circ} \mathrm{C}$ for $30 \mathrm{~min}$ or $8 \mathrm{~min}$ and washed with HEPES-buffer saline solution (HBSS). The measurements of $\mathrm{Ca}^{2+}$ signaling and LY uptake and HBSS preparation were performed as described previously $[17,40]$.

\section{Subcellular fractionations}

Fractionations of junctional and non-junctional $\mathrm{Cx} 43$ were prepared as described previously [43, 44] with some modifications. Briefly, the myocytes were flushed with cold PBS for two times and incubated in $100 \mu \mathrm{M}$ RIPA (CST) for 30 min on the ice and then scrapped and collected the suspension. The suspension was centrifuged at $10,000 \mathrm{rpm}$ for $5 \mathrm{~min}$ at $4{ }^{\circ} \mathrm{C}$, and collected the supernatants as nonjunctional fractions. The remaining cells pellet were resuspended, incubated after transient sonication in RIPA containing $2 \%$ Triton $\mathrm{X}-100$ and $0.4 \%$ SDS for $30 \mathrm{~min}$ at room temperature, and then centrifuged at $14,000 \mathrm{rpm}$ for $30 \mathrm{~min}$ at $4{ }^{\circ} \mathrm{C}$. The supernatants were the junctional proteins. GAPDH and $\beta$-actin were used as reference for non-junctional and junctional fraction separation, respectively.

\section{Co-immunoprecipitation and immunoblotting}

Cell protein was extracted with RIPA lysis buffer. The lysate was centrifuged at $12,000 \mathrm{rpm}$ for $15 \mathrm{~min}$ at $4{ }^{\circ} \mathrm{C}$, and the supernatant was used at once. Heart tissue samples were also prepared using RIPA buffer. Lysates were gently stirred by a mixer on ice for $30 \mathrm{~min}$, then were centrifuged at $15,000 \mathrm{rpm}$ for $15 \mathrm{~min}$ at $4{ }^{\circ} \mathrm{C}$. Protein concentration was measured by BCA assay kit (Thermo Scientific). Analysis of bands was performed and calculated using the chemiluminescence FluorChem FC3 system (ProteinSimple, San Jose, CA, USA). For immunoprecipitations, NRVMs or ventricle samples were extracted with mild lysis buffer and further analyzed as described previously $[17,40]$.

Antibodies used were as follows: rabbit anti-Fas, anti-NCad and anti-cytochrome C (Abcam), rabbit anti-pS282Cx43 and anti-pS279-Cx43 (Biobyt), rabbit anti-Cox IV, anti-p-p38 MAPK (Thr180/Tyr182), anti-p38 MAPK $\alpha$, anti-pS368-Cx43, anti-HA, anti-pS262-Cx43 and anti-Cx43 (Santa Cruz), and rabbit anti-ZO-1 (Proteintech), mouse anti-GAPDH and anti- $\beta$-actin (ZSGB-BIO), mouse antiTNFR1, anti-caspase-8 and anti-FADD (Santa Cruz), and goat anti-Cx43 (Acris) and anti-DR5 (Santa Cruz). All were used at the ratio of 1:1000, except for anti-FADD antibody at the ratio of 1:500.

\section{Statistical analysis}

All experiments were performed at least three times to assess reproducibility of the results. Data are presented as means \pm S.D., and were analyzed by Student's $t$ test for unpaired observations. For multiple groups, one-way ANOVA was performed with post Tukey adjustment when appropriate. $P<0.05$ was considered statistically significant.

Acknowledgements This work was supported by the National Natural Science Foundation $(81370339,81570206)$ and Scientific Research Key Program of Beijing Municipal Commission of Education (KZ201710025023).

Author contributions $\mathrm{YY}$ and $\mathrm{XY}$ contributed equally to the execution of most of the experiment and data analysis. JX planned and performed all $\mathrm{Ca}^{2+}$, Lucifer Yellow and cell death measurements, MC and ZS planned and performed immunofluorescence staining, TL planned and performed histochemistry, CW isolated and cultured cardiomyocytes and vascular smooth muscle cells, and HY generated and identified model animals. DL and YZ contributed to the design of the experiments, writing and data analysis of this manuscript. 


\section{Compliance with ethical standards}

Conflict of interest The authors declare that they have no conflict of interest.

Publisher's note: Springer Nature remains neutral with regard to jurisdictional claims in published maps and institutional affiliations.

\section{References}

1. Chouchani ET, Pell VR, Gaude E, Aksentijević D, Sundier SY, Robb EL, et al. Ischaemic accumulation of succinate controls reperfusion injury through mitochondrial ROS. Nature. 2014;515:431-5.

2. Eltzschig HK, Eckle T. Ischemia and reperfusion-from mechanism to translation. Nat Med. 2011;17:1391-401.

3. Chang KT, Cheng CF, King PC, Liu SY, Wang GS. CELF1 mediates connexin 43 mRNA degradation in dilated cardiomyopathy. Circ Res. 2017;121:1140-52.

4. Maguy A, Le Bouter S, Comtois P, Chartier D, Villeneuve L, Wakili $\mathrm{R}$, et al. Ion channel subunit expression changes in cardiac Purkinje fibers: a potential role in conduction abnormalities associated with congestive heart failure. Circ Res. 2009;104:1113-22.

5. Smyth JW, Hong TT, Gao D, Vogan JM, Jensen BC, Fong TS, et al. Limited forward trafficking of connexin 43 reduces cell-cell coupling in stressed human and mouse myocardium. J Clin Invest. 2010;120:266-79.

6. Chu M, Novak SM, Cover C, Wang AA, Chinyere IR, Juneman $\mathrm{EB}$, et al. Increased cardiac arrhythmogenesis associated with gap junction remodeling with upregulation of RNA-binding protein FXR1. Circ. 2018;137:605-18.

7. Britz-Cunningham SH, Shah MM, Zuppan CW, Fletcher WH. Mutations of the Connexin43 gap-junction gene in patients with heart malformations and defects of laterality. New Engl J Med. 1995;332:1323-29.

8. Gutstein DE, Morley GE, Tamaddon H, Vaidya D, Schneider $\mathrm{MD}$, Chen J, et al. Conduction slowing and sudden arrhythmic death in mice with cardiac-restricted inactivation of connexin 43. Circ Res. 2001;88:333-9.

9. Kalcheva N, Qu J, Sandeep N, Garcia L, Zhang J, Wang Z, et al. Gap junction remodeling and cardiac arrhythmogenesis in a murine model of oculodentodigital dysplasia. Proc Natl Acad Sci USA. 2007;104:20513-16.

10. Van Norstrand DW, Asimaki A, Rubinos C, Dolmatova E, Srinivas M, Tester DJ, et al. Connexin 43 mutation causes heterogeneous gap junction loss and sudden infant death. Circ. 2012;125:474-81.

11. Eckardt D, Kirchhoff S, Kim JS, Degen J, Theis M, Ott T, et al. Cardiomyocyte-restricted deletion of connexin 43 during mouse development. J Mol Cell Cardiol. 2006;41:963-71.

12. Van Rijen HV, Eckardt D, Degen J, Theis M, Ott T, Willecke K, et al. Slow conduction and enhanced anisotropy increase the propensity for ventricular tachyarrhythmias in adult mice with induced deletion of connexin43. Circ. 2004;109:1048-55.

13. Jeyaraman MM, Srisakuldee W, Nickel BE, Kardami E. Connexin43 phosphorylation and cytoprotection in the heart. Biochim Biophys Acta. 2012;18:2009-13.

14. Solan JL, Lampe PD. Connexin 43 phosphorylation: structural changes and biological effects. Biochem J. 2009;419:261-72.

15. Lampe PD, Cooper CD, King TJ, Burt JM. Analysis of connexin 43 phosphorylated at S325, S328 and S330 in normoxic and ischemic heart. J Cell Sci. 2006;119:3435-42.

16. Remo BF, Qu J, Volpicelli FM, Giovannone S, Shin D, Lader J, et al. Phosphatase-resistant gap junctions inhibit pathological remodeling and prevent arrhythmias. Circ Res. 2011;108: 1459-66.

17. Kang M, Na Lin, Chen Li, Meng Q, Zheng Y, Yan X, et al. Cx43 phosphorylation on S279/282 and intercellular communication are regulated by $\mathrm{IP}_{3} / \mathrm{IP}_{3}$ receptor signaling. Cell Commu Signal. 2014; $12: 58$.

18. Cheng J, Zhou T, Liu C, Shapiro JP, Brauer MJ, Kiefer MC, et al. Protection from Fas-mediated apoptosis by a soluble form of the Fas molecule. Science. 1994;263:1759-62.

19. Schalper KA, Palacios-Prado N, Orellana JA, Saez JC. Currently used methods for identification and characterization of hemichannels. Cell Commun Adhes. 2008;15:207-18.

20. Ma KT, Guan BC, Yang YQ, Nuttall AL, Jiang ZG. 2Aminoethoxydiphenyl borate blocks electrical coupling and inhibits voltage-gated $\mathrm{K}^{+}$channels in guinea pig arteriole cells. Am J Physiol-Heart C. 2011;300:H335-H46.

21. Rose BA, Force T, Wang Y. Mitogen-activated protein kinase signaling in the heart: angels versus demons in a heart-breaking tale. Physiol Rev. 2010;90:1507-46.

22. Martin ED, Bassi R, Marber MS. p38 MAPK in cardioprotectionare we there yet? Brit J Pharmacol. 2015;172:2101-13.

23. Bogoyevitch MA, Gullespie-Brown J, Ketterman A, Fuller S, Ben-Levy R, Ashworth A, et al. Stimulation of the stress-activated mitogen-activated protein kinase subfamilies in perfused heart: p38/RK mitogen-activated protein kinases and c-Jun N-terminal kinases are activated by ischemia reperfusion. Circ Res. 1996;79:62-73.

24. Hsu PL, Su BC, Kuok QY, Mo FE. Extracellular matrix protein CCN1 regulates cardiomyocyte apoptosis in mice with stressinduced cardiac injury. Cardiovasc Res. 2013;98:64-72.

25. Cook SA, Sugden PH, Clerk A. Activation of c-jun N-terminal kinases and p38-mitogen-activatedprotein kinases in human heart failure secondary to ischaemic heart disease. J Mol Cell Cardiol. 1999;31:1429-34.

26. Singal PK, Iliskovic N. Doxorubicin-induced cardiomyopathy. N Engl J Med. 1998;339:900-5.

27. Yeh ET, Bickford CL. Cardiovascular complications of cancer therapy: incidence, pathogenesis, diagnosis, and management. J Am Coll Cardiol. 2009;53:2231-47.

28. Decrock E, Vinken M, De Vuyst E, Krysko DV, D'Herde K, Vanhaecke T, et al. Connexin-related signaling in cell death: to live or let die? Cell Death Differ. 2009;16:524-36.

29. Srisakuldee W, Jeyaraman MM, Nickel BE, Tanguy S, Jiang Z-S, Kardami E. Phosphorylation of connexin 43 at serine 262 promotes a cardiac injury-resistant state. Cardiovasc Res. 2009;83:672-81.

30. Matsuyama D, Kawahara K. Oxidative stress-induced formation of a positive-feedback loop for the sustained activation of $\mathrm{p} 38$ MAPK leading to the loss of cell division in cardiomyocytes soon after birth. Basic Res Cardiol. 2011;106:815-28.

31. Schulz R, Gres P, Skyschally A, Duschin A, Belosjorow S, Konietzka I, et al. Ischemic preconditioning preserves connexin 43 phosphorylation during sustained ischemia in pig hearts in vivo. FASEB J. 2003;17:1355-7.

32. Porras A, Zuluaga S, Black E, Valladares A, Alvarez AM, Ambrosino C, et al. p38-mitogen-activated protein kinase sensitizes cells to apoptosis induced by different stimuli. Mol Bio Cell. 2004:15:922-33.

33. Engel FB, Hsieh PC, Lee RT, Keating MT. FGF1/p38 MAP kinase inhibitor therapy induces cardiomyocyte mitosis, reduces scarring, and rescues function after myocardial infarction. Proc Natl Acad Sci USA. 2006;103:15546-51.

34. Brichkina A, Nguyen NT, Baskar R, Wee S, Gunaratne $\mathrm{J}$, Robinson RC, et al. Proline isomerisation as a novel regulatory mechanism for p38MAPK activation and functions. Cell Death Differ. 2016;23:1592-601. 
35. Ge B, Gram H, Di Padova F, Huang B, New L, Ulevitch RJ, et al. MAPKK-independent activation of p38alpha mediated by TAB1dependent autophosphorylation of p38alpha. Science. 2002;295:1291-4.

36. De Nicola GF, Martin ED, Chaikuad A, Bassi R, Clark J, Martino $\mathrm{L}$, et al. Mechanism and consequence of the autoactivation of p38alpha mitogen-activated protein kinase promoted by TAB1. Nat Struct Mol Biol. 2013;20:1182-90.

37. Solan JL, Marquez-Rosado L, Sorgen PL, Thornton PJ, Gafken PR, Lampe PD. Phosphorylation of Cx43 at S365 is a gatekeeper event that changes the structure of $\mathrm{Cx} 43$ and prevents downregulation by PKC. J Cell Biol. 2007;179:1301-9.

38. Dunn CA, Lampe PD. Injury-triggered Akt phosphorylation of Cx43: a ZO-1-driven molecular switch that regulates gap junction size. J Cell Biol. 2014;127:455-64.

39. Axelsen LN, Stahlhut M, Mohammed S, Larsen BD, Nielsen MS, Holstein-Rathlou $\mathrm{NH}$, et al. Identification of ischemia-regulated phosphorylation sites in connexin43: a possible target for the antiarrhythmic peptide analogue rotigaptide (ZP123). J Mol Cell Cardiol. 2006;40:790-8.

40. Luo D, Yang D, Lan X, Li K, Li X, Chen J, et al. Nuclear $\mathrm{Ca}^{2+}$ sparks and waves mediated by $\mathrm{IP}_{3}$ receptors in neonatal rat cardiomyocytes. Cell Calcium. 2008;43:165-74.

41. Zinchuk V, Wu Y, Grossenbacher-Zinchuk O. Bridging the gap between qualitative and quantitative colocalization results in fluorescence microscopy studies. Sci Rep. 2013;3:1365.

42. Costes SV, Daelemans D, Cho EH, Dobbin Z, Pavlakis G, Lockett $\mathrm{S}$. Automatic and quantitative measurement of protein-protein colocalization in live cells. Biophys J. 2004;86:3993-4003.

43. Bruce AF, Rothery S, Dupont E, Severs NJ. Gap junction remodelling in human heart failure is associated with increased interaction of connexin43 with ZO-1. Cardiovasc Res. 2008;77:757-65.

44. Desplantez T, Grikscheit K, Thomas NM, Peters NS, Severs NJ, Dupont E. Relating specific connexin co-expression ratio to connexon composition and gap junction function. J Mol Cell Cardiol. 2015;89(Pt B):195-202. 